\title{
"HAVE A NICE DAY:)": SOCIAL INTERACTIONS WITH CUSTOMER SERVICE CHATBOTS
}

\author{
Stine Ordemann ${ }^{1}$, Marita Skjuve², Asbjørn Følstad ${ }^{2}$ and Cato Alexander Bjørkli ${ }^{1}$ \\ ${ }^{1}$ University of Oslo, Noway \\ ${ }^{2}$ SINTEF, Noway
}

\begin{abstract}
The use of chatbots in customer service is increasingly widespread. Although research investigating how users experience such chatbots exists, few studies have looked at how users actually interact with customer service chatbots. We lack knowledge on whether users participate in social interactions similar to what one would expect from a human-human interaction, or if they display different communication tactics. We carried out a study in which we asked 16 participants to interact with two customer service chatbots. The dialogues were then analysed to understand whether and how the users participated in social interaction. The results showed that most of the participants interact with chatbots in ways that resemble social interaction with humans, using full sentences as opposed to keywords, first and second person pronouns, and polite remarks. This shows that even highly task-oriented chatbots, such as chatbots for customer service, elicit various social responses from users and raises the question regarding whether one should design for social interaction with chatbots for customer service.
\end{abstract}

\section{KEYWORDS}

Customer Service Chatbots, Social Interaction, Dialogue Analysis

\section{INTRODUCTION}

Humans have a natural inclination to interact socially with digital technology (Nass et al., 1994). Think about it. Have you ever yelled at your computer when it was slow or praised your robot vacuum cleaner? Most of us can recall doing something similar.

This tendency to interact socially with non-living objects is a very interesting human behaviour that becomes even more relevant as technology grows more sophisticated. By social interaction with digital technology, we mean interaction that shares the characteristics of human interaction, such as the use of complete sentences rather than keywords, greetings, politeness markers (Nass et al., 1994), and personal pronouns (Brennan \& Ohaeri, 1994). Today, we use technology, such as chatbots, that can talk back to us in natural language, which arguably increases our tendency to interact socially with them.

Chatbots are software programs that can communicate with users in their natural language through text or voice (Følstad \& Brandtzaeg, 2020). Powered by artificial intelligence (AI) and machine learning, chatbots have emerged in various fields, supporting people with tasks ranging from banking inquiries (Følstad \& Skjuve, 2019) to workplace support (Meyer von Wolff et al., 2019) and health advice (Skjuve \& Brandtzæg, 2018). It is estimated that $85 \%$ of customer inquiries will be handled by chatbots in 2021 (Jovic, 2020). Successful interaction and adoption of such technology is therefore essential, and it is dependent on chatbot designs that facilitate effective and efficient interaction as well as a pleasant user experience.

While it is well known that users are inclined to engage in social interaction with chatbots developed for social purposes, such as Cleverbot (Hill et al., 2015) and Replika (Ta et al., 2020), there is a lack of knowledge concerning whether and how users interact socially with more task-oriented chatbots, such as chatbots for customer service.

Customer service chatbots are not typically designed for the purpose of having complex social interactions with the user, but rather to interpret a user request, often in the form of a problem that needs to be solved, and to provide the information to solve this problem (Følstad et al., 2018). Such chatbots are therefore designed to 
handle briefer problem statements, rather than long sentences, especially when these contain multiple questions and social content, such as polite remarks intertwined.

It is necessary that users understand the limitations of customer service chatbots and, to some extent, adapt their way of interacting with them. While this might seem like a simple task for humans, it is important to remember that human communication abilities have evolved over years to interact with other humans, but may not have adapted to communicate well with artificial entities (Lee, 2009).

Few studies have investigated how users interact with customer service chatbots. The objective of this study is to address one important aspect of such interaction: whether and how users engage in social interaction with customer service chatbots. Understanding this is essential if one seeks to design customer service chatbots with which users are able to have successful interaction with in the future.

\section{BACKGROUND}

\subsection{The Social Design of Chatbots}

There is a trend in the chatbot community to strive towards creating the most humanlike chatbot as possible. The annual tournament, the Loebner Prize Turing Test, in which a judge decides if their conversational partner is of human or chatbot origin, is an example (Coniam, 2014). Similarly, in the yearly Alexa Prize competition, teams compete in designing Alexa skills that enable Alexa to interact socially with users for a prolonged period of time (Ram et al., 2018).

Chatbots, for customer service purposes, are also designed with different attributes to enhance their human likeness and facilitate rudimentary social interaction. Such attributes may contribute to the deception of users in the sense that they engender imprecise perceptions of the nature and capabilities of the chatbots (Luger \& Sellen, 2016). Such attributes may include the use of informal language, names, avatars (Araujo, 2018), and gender (McDonnell \& Baxter, 2019) —all of which seem to have several positive effects.

The use of avatars has, for instance, been found to heighten trust resilience in chatbots when conversational flows are abrupted (De Visser et al., 2016), and the use of genders in the avatars has been shown to increase user satisfaction (McDonnell \& Baxter, 2019).

The implementation of social cues influences users' perception of chatbots and facilitates the phenomenon of anthropomorphism (Araujo, 2018). Anthropomorphism refers to "attributing humanlike properties, characteristics, or mental states to real or imagined nonhuman agents and objects" (Epley et al., 2007, p. 865). Anthropomorphism has been found to positively relate to users' adoption of chatbots, especially for customers who seek social interaction (Sheehan et al., 2020).

Likewise, humanlike cues in the chatbot language and interface can generate a feeling of social presence, which is defined by Lee (2004, p. 37) as "a psychological state in which virtual (para authentic or artificial) social actors are experienced as actual social actors in either sensory or non-sensory ways.

Our tendency to approach machines and other non-living artifacts in a social way is well established within the research and is the core in the Computers-Are-Social-Actors (CASA) paradigm, which holds that humans tend to participate in the mindless application of social rules towards machines (Nass \& Moon, 2000) by, for instance, applying gender stereotyping based on gender cues in the computer (Nass et al., 1997) or engage in polite behaviour towards computers (Nass et al., 1999).

Nass and Moon (2000) asserted that users are explicitly aware that computers do not warrant social treatment because they are non-living entities, nor do the users believe that they are communicating with the programmer (Nass et al., 1994). It is, therefore, not difficult to imagine that users would be inclined to interact socially towards a customer service chatbot that exhibits more humanlike cues than a computer, even though this type of chatbot is highly task-oriented. Research on customer service chatbots has, however, tended to focus more on users' experiences and perceptions of chatbots rather than the details of their actual interaction with these. 


\subsection{User Experience and Customer Service Chatbots}

Multiple studies have investigated how users perceive customer service chatbots. Zarouali et al. (2018), for instance, explored how different cognitive and affective factors influence users' attitudes towards the brand and, subsequently, their intentions to use and recommend a customer service chatbot in the future. They concluded that "how consumers think as well as how they feel about chatbots significantly influences the effectiveness of chatbot technology" ( $p, 496)$. Based on this, they recommend that designers should not only think about how to make chatbots useful and efficient but also how to facilitate feelings in the user by implementing jokes and emojis (Zarouali et al., 2018).

In a second study, Van den Broeck et al. (2019) explored how perceived helpfulness and usefulness affected the users' perceived intrusiveness when the chatbot tried to advertise something. Interestingly, they found that the more helpful and useful the chatbot was perceived as, the less intrusive the user experienced it to be.

Følstad and Skjuve (2019) interviewed 24 users of a customer service chatbot to uncover their motivations and experiences. They found that the chatbot's visual aspects and persona may not be that important in the real-world use of customer service chatbots. The participants were more concerned with the chatbot's ability to understand them and provide relevant information.

\subsection{Dialog Analysis of Customer Service Chatbot Conversations}

Only a few studies have investigated how users interact with customer service chatbots. Følstad (2020) analysed dialogues between users and a customer service chatbot. They found that a typical characteristic of unsuccessful conversations with the chatbot was long and complex sentences (Følstad, 2020). This study did not analyse the application of different social rules, such as politeness and the use of personal pronouns, which is typical in social interaction.

Kopp et al. (2005) investigated how people interacted with the chatbot Max, which had the role of a museum guide and could answer practical questions about the museum or participate in small talk. They analysed the dialog from interactions people have had with Max and concluded that people tended to participate in social interaction by saying "hello and goodbye" or using small talk. This study is highly relevant as it demonstrates that the users tended to engage in social interaction with this chatbot. However, Max is not a typical customer service chatbot. He is designed to provide practical information but also to facilitate small talk and display emotions (Kopp et al., 2005), which might contribute to the users interacting socially with him.

In a similar study, Jenkins et al. (2007) found that users displayed the same tendencies of saying hello and goodbye but that they used keywords rather than long sentences when interacting with the chatbot. Interestingly, they found that the participants tended to leave the keyword strategy after a few exchanges (Jenkins et al., 2007), which indicates that they may have drifted towards a more humanlike communication during the conversation. They concluded that "the users expect chatbot systems to behave and communicate like humans" (p. 83). One limitation with this study is that the chatbot they used was a prototype made over 10 years ago. Therefore, it might not be representative of the typical customer service chatbots in operation today.

\subsection{Summary and Current Study}

Few studies have investigated whether and how users interact socially towards customer service chatbots. Those that have either used chatbots with different purposes or prototypes of a customer service chatbot.

There is, therefore, a gap in the literature regarding how users interact with operative task-oriented customer service chatbots. This gap is potentially problematic in terms of existing research and practice. In research, this gap limits our knowledge concerning users' interactions with highly task-specific chatbots. In practice, this lack in knowledge leads to an insufficient knowledge base on which to make design decisions. This paper seeks to fill this gap by answering the following research question:

RQ: Whether and how may users' interactions with highly task-oriented chatbots for customer service be characterized as social interaction? 


\section{METHOD}

\subsection{Recruitment and Sample}

The study was conducted between November 11 and December 6, 2019. Sixteen participants were recruited through a convenience sample (Robinson, 2014). Individuals with a computer science education were excluded from the study. This was necessary due to their presumed knowledge of chatbot technology, which may have influenced their understanding of how such technology works and hence their way of interacting with them (Chen \& Wang, 2018).

The sample $(\mathrm{N}=16)$ consists of nine women $(56 \%)$ and seven men $(44 \%)$. The mean age was 27 , ranging from 21 to 47. Five participants (31\%) had achieved a master's degree, seven participants (43\%) had a bachelor's degree, and three (18\%) participants had finished one year of higher education. Most of the participants had some prior experience with chatbots.

\subsection{Data Collection}

We conducted a laboratory study in which the participants were asked to carry out a task developed to facilitate interactions with two different customer service chatbots.

\subsubsection{Choice of chatbots}

Two chatbots were used in the present study to facilitate more extensive interactions during the sessions. The chatbots were chosen based on similarity. Chatbots A and B were developed by the same company and based on the same technology. The interface of Chatbots A and B were similar in several ways. For example, they welcomed the users and provided a short introduction for interaction guidance. The first interaction was based on natural language, afterwards both natural language and predefined answer alternatives (presented as buttons) guided the conversations. When the chatbots were unable to answer a question correctly or interpret the user's intent, both presented the opportunity of talking to a human customer service agent, although only one of the chatbots provided this option within the same chat interface. The main visual differences between the two chatbots were gender and avatar. Chatbot A used a female avatar, while Chatbot B had a genderless robot avatar.

\subsubsection{Task Development}

We developed a task to guide the interaction, with the objective of creating a realistic context for the users. This has been done successfully in previous chatbot research (Chen \& Wang, 2018). In the task, the participants were instructed to find general information about mortgages. If the chatbots suggested that the participants enter a website for further information, they were asked to continue with the task of conversing with the chatbots. The task was relatively open-ended, with few requirements to make participants choose an interaction style that came naturally to them.

\subsubsection{Procedure}

The participants were given the task and provided with a computer. The two chatbots and screen capture videos were pre-prepared on the computer. The sequence of presentation of the chatbots was alternated to control for order effects. After completing their chatbot interaction, each participant was asked to report on how realistic they perceived the task to be (scores from one to five on a numbered scale). The participants were encouraged to talk out loud during their interactions, and interviews were conducted afterwards. This data is not included here.

\section{ANALYSIS}

The first step in the analysis was to develop an appropriate coding procedure, which was done by the first and third authors. The procedure included definitions to guide the analysis process. 
As noted previously, social interaction can include a variety of different behaviours ranging from small talk to the use of gender stereotypes when interacting with digital technology. There is no agreed-upon view on what constitutes social interaction with text-based chatbots, but we looked for the following aspects of the dialogues in evaluating the presence of social interaction.

\subsection{Application of Social Rules}

Social interaction with chatbots can be characterised by the use of longer or more complete sentences. While it is challenging to set up rules for what constitutes a complete or longer sentence, we discriminated between sentences and keyword tactics as follows: A sentence was defined as the use of four or more words in one message, while keyword tactics was defined as the use of three or fewer single words in one message.

\subsection{Use of Complete Sentences or Keyword Tactics}

The application of social rules or markers is a useful indicator of users' social interaction with chatbots. We therefore found the following to be particularly relevant: the use of first- and second-person pronouns and polite remarks. This is in line with Brennan and Ohaeri (1994), who defined an anthropomorphic sentence towards a computer agent as consisting of a first-person pronoun. They also found a higher use of second-person pronouns and polite remarks towards computers, indicating social interaction with the computer.

Once the coding procedure was established, the first author analysed the participants' dialogues. The coding was conducted in Excel, and this process was repeated three times to check for omissions in classification. A few omissions were found in the first analysis and corrected.

\section{RESULTS}

\subsection{Descriptive Statistics}

The participants spent more time with Chatbot $\mathrm{A}(\mathrm{M}=2.62 \mathrm{~min}, \mathrm{SD}=0.98 \mathrm{~min})$ than Chatbot $\mathrm{B}(\mathrm{M}=1.74$ $\min , \mathrm{SD}=0.51 \mathrm{~min})$, and a little over four minutes collectively with both chatbots $(\mathrm{M}=4.25 \mathrm{~min}, \mathrm{SD}=1.20$ $\min )$. Most participants perceived the task as representative of their natural usage $(\mathrm{M}=4.25, \mathrm{SD}=1.00)$.

\subsection{Language Analysis of the Chatbot Dialogue}

The purpose of the language analysis was to evaluate whether and how the users interacted socially with a customer service chatbot. The participants sent a total of 229 messages to the two chatbots, and the messages were analysed according to the following categories: (1) use of keyword vs. sentences, (2) addressing the chatbot with personal pronouns, and (3) polite remarks. Table 1 gives an overview of sample statements written in interaction with the chatbots, which are representative of the overall sample in each category, while Table 2 shows the prevalence of each category.

\subsection{Prevalence of Keywords or Sentences}

The participants seemed to prefer using complete sentences, such as "I want to apply for a mortgage. Can you give me information about this?", rather than just writing one word, such as "loan".

Out of the 229 messages, a total of 58 reflected a keyword tactic (defined as three words or fewer). The remaining 171 messages were comprised of complete sentences (four words or more). Only two out of 16 participants used a keyword tactic in their interaction in more than half of their messages.

\subsection{Prevalence of Personal Pronouns}

The use of personal pronouns was prevalent in the dataset. The participants typically referred to themselves when asking a question, such as "Do I need a permanent job to get my first mortgage?" (P 8). Out of the 229 messages, a total of 104 messages contained Norwegian first- and second-person pronouns, and seven out of the 16 participants used first- and second-person pronouns in half of their messages or more. 


\subsection{Prevalence of Polite Remarks}

Politeness was also used by almost all the participants. This typically included saying hello or goodbye to the chatbot and using phrases such as "Have a nice day :)" (P 13). Twenty-seven out of 229 messages contained politeness, and 12 out of 16 displayed some form of politeness towards the chatbot.

Table 1. Written examples of different language categories

\begin{tabular}{ll}
\hline Category & Statements \\
\hline Sentences & "What do you need to know when applying for a mortgage?" (P 5) \\
& "If taking out a mortgage for a residence worth 5 million, how much \\
& equity is needed?" $(P$ 6) \\
& "My partner bought an apartment last year." $(P 4)$ \\
& "Does bsu count the same as other equity?" $(P$ 14)
\end{tabular}

Note. Numbers in the parentheses reflect the participant's ID.

Table 2. Prevalence of Language Categories for Each Participant's Messages

\begin{tabular}{cccc}
\hline $\begin{array}{c}\text { Participant's } \\
\text { ID }\end{array}$ & $\begin{array}{c}\text { Use of } \\
\text { Keywords }\end{array}$ & $\begin{array}{c}\text { Use of Personal } \\
\text { Pronouns }\end{array}$ & $\begin{array}{c}\text { Use of Polite } \\
\text { Remarks }\end{array}$ \\
\hline 1 & $12 \%$ & $50 \%$ & $25 \%$ \\
2 & $25 \%$ & $61 \%$ & - \\
3 & $11 \%$ & $77 \%$ & - \\
4 & $7 \%$ & $66 \%$ & - \\
5 & $12 \%$ & $50 \%$ & $25 \%$ \\
6 & $7 \%$ & $30 \%$ & $15 \%$ \\
7 & $15 \%$ & $61 \%$ & $15 \%$ \\
8 & - & $44 \%$ & - \\
9 & $31 \%$ & $57 \%$ & $5 \%$ \\
10 & $4 \%$ & $41 \%$ & - \\
11 & - & $41 \%$ & $16 \%$ \\
12 & $43 \%$ & $43 \%$ & $21 \%$ \\
13 & $36 \%$ & $42 \%$ & $31 \%$ \\
14 & $20 \%$ & $40 \%$ & $13 \%$ \\
15 & $62 \%$ & $25 \%$ & $6 \%$ \\
16 & $68 \%$ & $18 \%$ & $12 \%$ \\
Total & $25 \%$ & $45 \%$ & $11 \%$ \\
\hline
\end{tabular}

Note. The percentage is calculated based on the total written messages per participant with both chatbots. Empty spaces (-) represent a lack of applied category. 


\section{DISCUSSION}

The analysis of the chatbot dialogues showed that the users indeed seemed to participate in social interaction with the customer service chatbots. The participants generally preferred the use of longer sentences and engaged less in keyword tactics. The results also demonstrated the application of social rules by all participants in some or most of their interaction, such as the use of personal pronouns and polite remarks.

The participants were fully aware that they were interacting with task-oriented chatbots, but they still seemed to prefer to engage in social interaction. This is in line with other research (Jenkins et al., 2007; Kopp et al., 2005) and exemplifies what Nass and Moon (2000) call ethopoeia, a "direct response to an entity as human while knowing that the entity does not warrant human treatment or attribution" (p. 94).

Social scripts are assumed to be the underlying explanation of this phenomenon. Scripts are mental processes that help us understand how to behave in various situations (Abelson, 1981). Lee and See (2004) suggest that human cognition is "tricked" by evolutionary-based dispositions, which can explain why social scripts are applied in this novel context.

This explanation can also be related to the concept of overlearned social behaviour, which is so ingrained and automatic that it occurs without conscious attention (Nass \& Moon, 2000). The use of natural language in chatbots may trigger such overlearned responses, regardless of the purpose of the chatbot. This tendency seems to be prevalent in the Jenkins et al. (2007) study as well, in which the users started off with a keyword tactic, but then switched to longer sentences during the interaction. Hence, it might require very little for the users to fall back to what is most familiar-social interaction.

However, this does not mean that communication with chatbots is identical to communication with humans. One should be cautious when interpreting the present results, as it seems unlikely that the participants would have displayed identical communication in customer service chats operated by humans. This is supported by the Hill et al. (2015) findings on human communication with chatbots and humans, in which the respective interactions exhibited a significant difference in many attributes. Therefore, a nuanced and plausible explanation is that people are generally aware of these distinctions and adapt accordingly, without fully abandoning a social way of interacting.

\subsection{Should We Design for Social Interactions with Customer Service Chatbots?}

In light of these findings, an important question to consider is whether to strive towards the promotion of social interaction when designing customer service chatbots, even when they are highly task oriented. This form of interaction is more complex, and the chatbot needs to be quite sophisticated in order to successfully handle these dialogues.

One approach to this issue, which seems to be the default way today, is to try to improve the chatbots' capabilities. Another way, however, is to design chatbots in a way that does not trigger social interaction to an extent that is difficult for the chatbot to handle. In the two chatbots used in this study, this latter approach was already pursued to some extent by limiting the number of characters the user could include in each message. However, other approaches may also be relevant. This might be a more tedious task for the designer, but it may also result in more successful human-chatbot conversations. If one seeks to design "against" social interaction, it might be worth trying the following:

- Welcome dear customer. Customer service chatbots tend to welcome the user in a social way, using long and complex sentences, and sometimes emojis. One of the chatbots used in this study, for instance, says the following: "Hey you! My name is [chatbot name] and I am [company name] chatbot (:) What can I help you with? This is an open chat, so please do not write anything personal like your name, birth number or health information." While this communication is welcoming, it might also reinforce the same social behaviour from the user, such as "I want to apply for a mortgage. Can you give me information about this?" Previous research has shown that task-oriented chatbots often struggle to understand such inquiries (Toxtli et al., 2018). It might, therefore, be interesting to see what happens if the chatbot were to greet the user more straightforwardly, such as: "Hello, what can I help you with."

- I am a chatbot. The designers of customer service chatbots often try to mitigate the user treating the chatbot as if it were human by explaining that it is artificial and that the user should ask very specific questions with short sentences. However, it could be that this too facilitates social interaction. The long "I'm a chatbot introduction" might not be fully comprehended by the user and interpreted more as 
social interaction from the chatbot, which is then reciprocated. Some may argue that making the user aware of the chatbot being a chatbot is important to set the right expectations, but we currently do not even know how the user draws this conclusion. The user might understand this based on the chatbot's fast response, because the chatbot says so, or from experience or the webpage, and so on. Therefore, having the chatbot explain this might be counterproductive.

\section{LIMITATIONS AND FUTURE WORK}

This study should be considered as a starting point for future research and explorations, as it has several limitations. First, the sample is small, and future studies should analyse a larger set of dialogues. Second, while the participants rated the task as highly realistic, it is still a laboratory study. Research analysing real dialogue is therefore needed. Third, we carried out a simple and somewhat superficial analysis. It would therefore be interesting to conduct similar studies but with more extensive analysis to see if other ways of participating in social interaction are uncovered. Fourth, we did not analyse whether there is a relationship between social interaction and the successful handling of user requests. However, there are reasons to suspect that there is a relationship between these two aspects, and future research should therefore investigate how social interaction affects the chatbot's ability to successfully handle the user's requests.

\section{REFERENCES}

Abelson, R. P. (1981). Psychological status of the script concept. American Psychologist, 36(7), 715.

Araujo, T. (2018). Living up to the chatbot hype: The influence of anthropomorphic design cues and communicative agency framing on conversational agent and company perceptions. Computers in Human Behavior, 85, 183-189. doi:10.1016/j.chb.2018.03.051

Brennan, S. E., \& Ohaeri, J. O. (1994). Effects of message style on users' attributions toward agents. Paper presented at the Conference Companion on Human Factors in Computing Systems.

Chen, M.-L., \& Wang, H.-C. (2018). How personal experience and technical knowledge affect using conversational agents. Paper presented at the Proceedings of the 23rd International Conference on Intelligent User Interfaces Companion, Tokyo.

Coniam, D. (2014). The linguistic accuracy of chatbots: Usability from an ESL perspective. Text \& Talk, 34(5), 545-567. doi:10.1515/text-2014-0018

De Visser, E. J., Monfort, S. S., McKendrick, R., Smith, M. A., McKnight, P. E., Krueger, F., \& Parasuraman, R. (2016). Almost human: Anthropomorphism increases trust resilience in cognitive agents. Journal of Experimental Psychology: Applied, 22(3), 331. doi:10.1037/xap0000092

Epley, N., Waytz, A., \& Cacioppo, J. T. (2007). On seeing human: A three-factor theory of anthropomorphism. Psychological review, 114(4), 864. doi:10.1037/0033-295X.114.4.864

Følstad, A. (2020). Improving Conversations: Lessons Learnt from Manual Analysis of Chatbot Dialogues. Paper presented at the Chatbot Research and Design: Third International Workshop, CONVERSATIONS 2019, Amsterdam, The Netherlands, November 19-20, 2019, Revised Selected Papers.

Følstad, A., \& Brandtzaeg, P. B. (2020). Users' experiences with chatbots: Findings from a questionnaire study. Quality and User Experience, 5, 1-14.

Følstad, A., \& Skjuve, M. (2019). Chatbots for customer service: User experience and motivation. Paper presented at the Proceedings of the 1st International Conference on Conversational User Interfaces.

Følstad, A., Skjuve, M., \& Brandtzaeg, P. B. (2018). Different chatbots for different purposes: Twards a typology of chatbots to understand interaction design. Paper presented at the International Conference on Internet Science.

Hill, J., Ford, W. R., \& Farreras, I. G. (2015). Real conversations with artificial intelligence: A comparison between human-human online conversations and human-chatbot conversations. Computers in Human Behavior, 49, $245-250$.

Jenkins, M.-C., Churchill, R., Cox, S., \& Smith, D. (2007). Analysis of user interaction with service oriented chatbot systems. Paper presented at the International Conference on Human-Computer Interaction.

Jovic, D. (2020). The future is now - 37 fascinating chatbot statistics. Retrieved from https://www.smallbizgenius.net/bythe-numbers/chatbot-statistics/\#gref 
Kopp, S., Gesellensetter, L., Krämer, N. C., \& Wachsmuth, I. (2005). A conversational agent as museum guide-design and evaluation of a real-world application. Paper presented at the International Workshop on Intelligent Virtual Agents.

Lee, J. D., \& See, K. A. (2004). Trust in automation: Designing for appropriate reliance. Human factors, 46(1), 50-80.

Lee, K. M. (2004). Presence, explicated. Communication Theory, 14(1), 27-50. doi:10.1111/j.1468-2885.2004.tb00302.x

Lee, K. M. (2009). Presence Theory. In S. W. Littlejohn \& K. Foss, A (Eds.), Encyclopedia of communication theory (pp. 794-796). SAGE Publications. Retrieved from https://sk-sagepubcom.ezproxy.uio.no/reference/communicationtheory/n301.xml.

Luger, E., \& Sellen, A. (2016). "Like having a really bad PA"- The gulf between user expectation and experience of conversational agents. Paper presented at the Proceedings of the $2016 \mathrm{CHI}$ Conference on Human Factors in Computing Systems.

McDonnell, M., \& Baxter, D. (2019). Chatbots and gender stereotyping. Interacting with Computers, 31(2), 116-121. doi:10.1093/iwc/iwz007

Meyer von Wolff, R., Hobert, S., \& Schumann, M. (2019). How may I help you?- State of the art and open research questions for chatbots at the digital workplace. Paper presented at the Proceedings of the 52nd Hawaii International Conference on System Sciences.

Nass, C., \& Moon, Y. (2000). Machines and mindlessness: Social responses to computers. Journal of Social Issues, 56(1), 81-103.

Nass, C., Moon, Y., \& Carney, P. (1999). Are respondents polite to computers? Social desirability and direct responses to computers. Journal of Applied Social Psychology, 29(5), 1093-1110. doi:10.1111/j.1559-1816.1999.tb00142.x

Nass, C., Moon, Y., \& Green, N. (1997). Are machines gender neutral? Gender-stereotypic responses to computers with voices. Journal of Applied Social Psychology, 27(10), 864-876. doi:doi.org/10.1111/j.1559-1816.1997.tb00275.x

Nass, C., Steuer, J., \& Tauber, E. R. (1994). Computers are social actors. Paper presented at the Proceedings of the SIGCHI Conference on Human factors in Computing Systems.

Ram, A., Prasad, R., Khatri, C., Venkatesh, A., Gabriel, R., Liu, Q., ... Nagar, A. (2018). Conversational AI: The science behind the Alexa Prize. arXiv preprint arXiv:1801.03604.

Robinson, O. C. (2014). Sampling in interview-based qualitative research: A theoretical and practical guide. Qualitative Research in Psychology, 11(1), 25-41. doi:10.1080/14780887.2013.801543

Sheehan, B., Jin, H. S., \& Gottlieb, U. (2020). Customer service chatbots: Anthropomorphism and adoption. Journal of Business Research, 115, 14-24. doi:10.1016/j.jbusres.2020.04.030

Skjuve, M., \& Brandtzæg, P. B. (2018). Chatbots as a new user interface for providing health information to young people. In Y. Andersson, U. Dahlquist, \& J. Ohlsson (Eds.), Youth and news in a digital media environment-Nordic-Baltic perspectives. Nordicom. Retrieved from https://www.nordicom.gu.se/sv/system/tdf/publikationer-helapdf/youth_and_news_in_a_digital_media_environment.pdf?file $=1 \&$ type $=$ node $\&$ id $=39917 \&$ force $=0$.

Ta, V., Griffith, C., Boatfield, C., Wang, X., Civitello, M., Bader, H., . . Loggarakis, A. (2020). User experiences of social support from companion chatbots in everyday contexts: Thematic analysis. Journal of Medical Internet Research, 22(3), e16235.

Toxtli, C., Monroy-Hernández, A., \& Cranshaw, J. (2018). Understanding chatbot-mediated task management. Paper presented at the Proceedings of the 2018 CHI Conference on Human Factors in Computing Systems.

Van den Broeck, E., Zarouali, B., \& Poels, K. (2019). Chatbot advertising effectiveness: When does the message get through? Computers in Human Behavior, 98, 150-157.

Zarouali, B., Van den Broeck, E., Walrave, M., \& Poels, K. (2018). Predicting consumer responses to a chatbot on Facebook. Cyberpsychology, Behavior, and Social Networking, 21(8), 491-497. 